\title{
Efficacy of Prakriti specific Herbal Tea in Diabetes A Randomized controlled trial
}

\author{
Research Article
}

\section{Sapna Sehgali', Huddar VG ${ }^{2}$, Mangalagowri V Rao $^{3 *}$}

\author{
1. Medical Officer, ESIC Hospital, Manesar, Hariyana, \\ 2. Associate Professor, All India Institute of Ayurveda, New Delhi, \\ 3. Associate Professor, Department of Swasthavritta and Yoga, Faculty of Ayurveda, \\ Institute of Medical Sciences, Banaras Hindu University, Varanasi.
}

\begin{abstract}
Introduction: Prakriti has an influential role in diagnosis and management of any disease. Increasing pandemic of diabetes leads to early mortality and decreased quality of life due to severe complications and side effect of contemporary medicines. The present study reveals Prakriti specific combination of herbs in form of tea with antidiabetic effect and no side effects. Materials and methods: 115 patients were enrolled which was randomly distributed in 2 group. Group A was Prakriti specific Herbal tea trial group and Group B was control. Both subjective and objective parameters were assessed. Results:Highly significant results with $\mathrm{p}$ value $<0.0001$ were seen in Polyuria (Prabhutmutrata), Laziness (Alasya), Excess Sleep (Nidraadhikya), Dryness in Mouth(Gala Talu Shosha), Excessive Thirst (Ati Pipasa), with maximum improvement in Polyuria (Prabhutmutrata).Significant results were seen in Burning Sensation in hands and legs (Karpaddaha),Numbness in palm and foot (Karpadasuptata), Cramps (Pindikodweshtana). Highly significant results were seen in Fasting, Postprandial Blood sugar, HbA1c and urine fasting glucose was seen in Prakriti specific Herbal tea group with $\mathrm{p}$ value $<0.0001$. Maximum improvement were seen in Kapha Prakriti patients with p value $<0.0001$ followed by Vata Prakriti patients. Vata and Kapha Herbal Tea found equally effective.Conclusion: Prakriti specific Herbal Tea is a better option of Diabetes Mellitus with high efficacy.
\end{abstract}

Key Words: Prakriti, Diabetes, Herbal tea, Prameha.

\section{Introduction}

Diabetes Mellitus has emerged as an important public health problem globally. It is estimated that global economic burden will increase from U.S. \$1.3 trillion $(95 \%$ CI $1.3-1.4)$ in 2015 to $\$ 2.2$ trillion $(2.2-$ $2.3)$ in the baseline, $\$ 2.5$ trillion (2.4-2.6) in the past trends, and \$2.1 trillion (2.1-2.2) in the present scenarios by 2030 (1) Diabetes Mellitus can be correlated with Prameha/Madhumeha due to similarity in their aetiology and characteristic features.In Ayurveda, Prameha is a Tridoshaja Vyadhi (2)with genetic predisposition(3) as well as improper diet and lifestyle Progression of Prameha to Madhumeha (Ojomeha) occurs with progression of time leading to the loss of Ojus (the essence part of all body tissues).The Ayurvedic management principles consist of Samshodhana (Purification), Samshamana (alleviation therapy), Nidana parivarjana (avoiding causative factors) in the form of Aahara (Diet) and Vihara (Lifestyle) is based on Dashavidha Parikshya

\section{* Corresponding Author:}

\section{Mangalagowri V Rao}

Associate Professor,

Dept. of Swasthavritta and Yoga, Faculty of Ayurveda, Institute of Medical Sciences,

Banaras Hindu University, Varanasi-221005, India

Email Id: mangowri@gmail.com
Bhavas( Ten diagnostic factors). Prakriti has key role in incidence and management of disease, which is described as first and most important factor in Parikshya Bhavas. Thus Prakriti based diet and management is highly essential for better results. In the present day lifestyle, tea forms the most important component in diet of most individuals. So the Prakriti based Herbal tea, which includes kitchen herbs and spices as major ingredients with proven antidiabetic effects, would benefit the individuals and can be incorporated in the daily routine easily. As per studies only $41 \%$ of patients on anti-diabetic therapy had optimal glycaemic control with conventional management.(4) As holistic approach the study was conducted to give emphasis on alleviation of disease without any adverse effect. Ingredients included in Prakriti specific Herbal tea (Kapha, Vata, Pitta) are suitable for specific Prakriti and pacify the Vitiated dosha of Madhumeha by rectifying the Agni especially Dhatvagni resulting in proper metabolism and control of blood sugar.

\section{Aims and objectives}

- To evaluate the efficacy of Prakriti specific Herbal Tea (Phanta) along with ongoing standard hypoglycaemic drugs in patients with Diabetes Mellitus type 2.

- To compare the results with the existing generalised Ayurvedic diabetic diet. 


\section{Materials and Methods}

The present study was conducted in two phases. Preliminary phase and clinical trial phase. Preliminary phase consist of identification and procurement of herbs, preparation of herbal tea and Analytical study of herbal tea.List of herbs used in three Prakriti specific herbal tea are given below.

\section{Ingredients of Vata Herbal tea for Vatapradhana Prakriti}

Sunthi (Zingiber officinale Rosc) $500 \mathrm{mg}$, Tulasi (Ocimum sanctum Linn) $500 \mathrm{mg}$, Ela (Elaterria cardamomum Maton) $500 \mathrm{mg}$, Lavanga (Syzygium aromaticumLinn. $250 \mathrm{mg}$, Mishreya (Foenieulum vulgare Mill.) $500 \mathrm{mg}$, Patra (Cinnamomum tamala Nees \& Eberm) $500 \mathrm{mg}$, Jatiphala (Myristica fragrans Houtt) $100 \mathrm{mg}$, Tagar (Valeriana wallichii DC) $250 \mathrm{mg}$, Meshashringi (Gymnema sylvestre $R \mathrm{Br}$ ) $2.5 \mathrm{gm}$, Jeerak (Cuminum cyminum Linn.) $500 \mathrm{mg}$.

\section{Ingredients of Kapha Herbal tea for Kaphapradhana Prakriti}

Twak (Cinnamomum zeylanicum Breyn.) 1 gm, Brihatela (Amomum subulatum Roxb) $250 \mathrm{mg}$, Bilwa Patra (Aegle marmelos Corr.) $250 \mathrm{mg}$, Mishreya (Foenieulum vulgare Mill.)250 $\mathrm{mg}$, Jatiphala (Myristica fragrans Houtt) $\quad 100 \mathrm{mg}$, Sunthi (Zingiber officinale Rosc.) $250 \mathrm{mg}$, Meshashringi (Gymnema sylvestre R Br.) 2.5 gm, Jambu (Syzygium cumini Linn) $500 \mathrm{mg}$, Lavang (Syzygium aromaticumLinn.) $200 \mathrm{mg}$, Tulsi (Ocimum sanctum Linn.) $500 \mathrm{mg}$, Maricha (Piper nigrum Linn.) $100 \mathrm{mg}$

\section{Ingredients of Pitta Herbal tea for Pittapradhana Prakriti}

Dhanyaka (Coriander sativum Linn.) $500 \mathrm{mg}$, Haridra (Curcuma longa Linn.) $500 \mathrm{mg}$, Mishreya (Foenieulum vulgare Mill.) $500 \mathrm{mg}$, Ela (Elaterria cardamomum Maton) $500 \mathrm{mg}$, Asana (Pterocarpus marsupium Roxb.) $2.5 \mathrm{gm}$, Sunthi (Zingiber officinale Rosc.) $500 \mathrm{mg}$, Tulasi (Ocimum sanctum Linn) $500 \mathrm{mg}$, Udumbara (Ficus glomerata Roxb.)1 gm

\section{Manufacturing of Prakriti specific Herbal Tea powder}

Preparation of Prakriti specific Herbal tea powder was done by Multani pharmaceuticals with authenticated ingredients under the supervision of competent authority.

\section{Clinical study}

Present study was conducted at Kayachikitsa OPD and Swasthavritta OPD in All India Institute of Ayurveda, Delhi. Approval from Institutional Review Board was taken followed by Ethical clearance from Institutional Ethical Committee with number IEC-AIIA 2017/PG-37. Further, it was registered in Clinical Trial Registry of India with number CTRI/2018/03/012483 on March 2018. About 115 patients of Prameha (Diabetes Mellitus) belonging to 30-60 yrs. of age group and both gender were selected based on fulfilment of diagnostic criteria and randomly allocated in 2 groups after getting the informed consent. The Prakriti of the individual subject was evaluated based on Prakriti, Prototype Prakriti Analysis Tool (PPAT) (5).

\section{Inclusion Criteria}

FPG $\geq 126 \mathrm{mg} / \mathrm{DL}(7.0 \mathrm{mmol} / \mathrm{L})-$-Fasting is defined as no caloric intake for $\geq 8$ hours to $220 \mathrm{mg} / \mathrm{dl}$, $2-\mathrm{hr}$ PG $\geq 200(11.1 \mathrm{mmol} / \mathrm{L})$ to $300 \mathrm{mg} / \mathrm{dl}$, both newly and previously diagnosed diabetes mellitus patients with less than 7 years

\section{Exclusion Criteria}

Patients having serious cardiac disorders like cardiac failure, patients having major illness like IDDM, patients having a history of untreated thyroid disorders, pregnant females and lactating mothers, renal insufficiency.

\section{Intervention}

In Group A patients were administered with 80-100ml of Prakriti specific Herbal tea twice a day at $40^{\circ} \mathrm{C}-50^{\circ} \mathrm{C}$ along with Pathyaapthya advice with their ongoing treatment for diabetes Mellitus for the duration of 3 months. The patients in group B were given only Pathyaapathya advice and lukewarm water at $40^{\circ} \mathrm{C}-50^{\circ} \mathrm{Calongwith}$ their ongoing treatment for Diabetes Mellitus for the duration of 3 months.

\section{Preparation of Prakriti specific Herbal Tea (Phanta)}

$6 \mathrm{gm}$ of Herbal Tea powder was mixed in 80-100 $\mathrm{ml}$ of water followed by roll boiling method. Herbal tea was administered in the morning and evening. The dosage of tea i.e. $80-100 \mathrm{ml}$ bid is as per general tea consumption amount.

Total Monitoring period $=3$ months with an interval of 1 month

\section{Assessment parameters}

Objective parameters:

CBC, Serum Cholesterol, Serum Triglyceride, Fasting Blood Glucose, Postprandial Blood Glucose, and $\mathrm{HbAlc}$.

Subjective parameters:

Prabhutmutrata (Polyuria), Avilamutrata (Turbidity In Urine), Karpaddaha (Burning Sensation In Hands And Legs), Madhuryamasya (Sweet Taste In The Mouth), Alasya (Laziness), Nidraadhikya (Excess Sleep), Atikshudha (Excessive Hunger), Karapadasuptata (Numbness In Palm And Foot), Gala Talu Shosha (Dryness In Mouth), Pindikodveshtana (Cramps), Ati Pipasa (Excessive Thirst), Swedadhikya (Excess Perspiration), assessed by grading criteria (6).

\section{Statistical analysis}

Paired $t$ test, unpaired $t$ test, one way ANOVA and repeated measured ANOVA was applied by using Graph Pad Prism version 5.03. 


\section{Observations and results}

Out of 115 Patients 20 patients in Group A and 20 patients from Group B dropped out at different follow-ups. 40 in group A and 35 in group B have completed the study.

In the present study Maximum patients were females $(58.2 \%)$ belonging to 40 to 60 years of age $(72.6 \%)$. Most of the patients had sedentary habits $(54.7 \%)$ and came under middle income group (62\%). BMI range in maximum patients $(40 \%)$ was 25.0-29.9.45.2\% of patients had positive family history for diabetes. About $10 \%$ patients consumed alcohol occasionally, and about $8 \%$ patients were smokers and $2 \%$ chewed tobacco.

Diet history revealed mixed diet $(58.2 \%)$ practice in majority. 37.3\% patients consumed Guru guna predominant diet, 22.6\% with Snighdha Ahara and $33.9 \%$ patients took. Lavana Rasa predominant diet. About $57 \%$ patients consumed rice as a staple diet, $43.4 \%$ taking Red gram. Maximum patients (46.9\%) were regularly taking banana. $69.5 \%$ of patients were taking milk regularly, $59 \%$ were taking curd regularly.
$56.5 \%$ of patients were consuming mustard oil and $66.9 \%$ of patients were consuming baked items/fast food. Most patients $(71.3 \%)$ consumed meals at irregular time.

The study data reveals that maximum number of patients i.e. $33.9 \%$ had Kapha Pradhana Vata Anubandh Prakriti, followed by $20.8 \%$ of Vata Pradhana Kapha Anubandhan, 17.3\% Vata Pradhan Pittaja Anubandh, 20\% Kapha Pradhan Pittaja Anubandh, 10\% patients were of Pittapradhana Vataja Anubandh Prakriti and the least number of patients had Pittapradhana Vataja Anubandh .Prakriti i.e. $4.3 \%$.Observations on various clinical features showed that the main presenting complaints of the patients were Polyuria (Prabhutmutrata), Burning Sensation In Hands And Legs (Karpaddaha), Laziness (Alasya), Excessive Hunger (Atikshudha), Numbness In Palm And Foot (Karapadasuptata), Dryness In Mouth (Gala Talu Shosha), Cramps (Pindikodveshtana), Excessive Thirst (AtiPipasa). It was also observed Polyuria (Prabhutmutrata), Laziness (Alasya) and Excessive Thirst (Atipipasa) were seen in 70 percent of patients.

Intra and Inter group cpmparison of Subjective Parameters

Table 1: Effect of Prakriti specific Herbal Tea treatment (Group A) in subjective parameters

\begin{tabular}{|c|c|c|c|c|}
\hline Subjective parameters & BT & AT & $\begin{array}{l}\text { P value } \\
\text { (t test) }\end{array}$ & $\begin{array}{l}\text { Percentage } \\
\text { of relief }\end{array}$ \\
\hline Prabhutmutrata (Polyuria) & $2.744 \pm 1.117$ & $0.6103 \pm 0.5916$ & $<0.0001$ & $76 \%$ \\
\hline Avilamutrata (Turbidity In Urine) & $0.976 \pm 0.871$ & $0.9108 \pm 0.4328$ & 0.0532 & $0.06 \%$ \\
\hline $\begin{array}{l}\text { Karpaddaha (Burning Sensation In } \\
\text { Hands And Legs) }\end{array}$ & $1.063 \pm 1.8012$ & $0.8 \pm 0.5214$ & 0.034 & $24 \%$ \\
\hline $\begin{array}{l}\text { Madhuryamasya (Sweet Taste In The } \\
\text { Mouth) }\end{array}$ & $1.9876 \pm 1.7654$ & $0.7691 \pm 0.4328$ & 0.069 & $61 \%$ \\
\hline Alasya (Laziness) & $3.694 \pm 2.7319$ & $1.0621 \pm 0.9761$ & 0.0003 & $68 \%$ \\
\hline Nidraadhikya (Excess Sleep) & $3.102 \pm 2.430$ & $1.872 \pm 1.209$ & 0.0012 & $41 \%$ \\
\hline Atikshudha (Excessive Hunger) & $0.925 \pm 1.163$ & $0.313 \pm 0.987$ & 0.034 & $65 \%$ \\
\hline $\begin{array}{l}\text { Karapadasuptata (Numbness In Palm } \\
\text { And Foot) }\end{array}$ & $2.0931 \pm 1.9724$ & $1.212 \pm 0.921$ & 0.0231 & $42 \%$ \\
\hline Gala Talu Shosha (Dryness In Mouth) & $2.1534 \pm 2.0198$ & $0.9213 \pm 0.8162$ & 0.0009 & $57 \%$ \\
\hline Pindikodveshtana (Cramps) & $1.9501 \pm 2.0198$ & $0.9213 \pm 0.8162$ & 0.047 & $52 \%$ \\
\hline Ati Pipasa (Excessive Thirst) & $0.925 \pm 1.163$ & $0.420 \pm 0.987$ & 0.002 & $55 \%$ \\
\hline Swedadhikya (Excess Perspiration) & $3.067 \pm 2.9540$ & $1.6120 \pm 0.7132$ & $<0.0001$ & $47 \%$ \\
\hline
\end{tabular}

Table 2: Effect on control Group B on subjective parameters

\begin{tabular}{|l|l|l|l|l|}
\hline Subjective parameters & BT & AT & $\begin{array}{l}\text { P value } \\
\text { (t test) }\end{array}$ & $\begin{array}{l}\text { Percentage } \\
\text { of relief }\end{array}$ \\
\hline $\begin{array}{l}\text { Prabhutmutrata (Polyuria } \\
\text { Avilamutrata (Turbidity In Urine) }\end{array}$ & $1.983 \pm 1.093$ & $1.054 \pm 0.9324$ & 0.0034 & $47 \%$ \\
\hline $\begin{array}{l}\text { Karpaddaha (Burning Sensation In Hands And } \\
\text { Legs) }\end{array}$ & $1.165 \pm 1.076$ & $1.143 \pm 0.9324$ & 0.0671 & $0.013 \%$ \\
\hline $\begin{array}{l}\text { Madhuryamasya (Sweet Taste In The Mouth) } \\
\text { Alasya (Laziness) }\end{array}$ & $0.9231 \pm 0.8071$ & $0.4359 \pm 0.5980$ & 0.0431 & $52 \%$ \\
\hline $\begin{array}{l}\text { Nidraadhikya (Excess Sleep) } \\
\text { Atikshudha (Excessive Hunger) }\end{array}$ & $3.4231 \pm 2.9990$ & $2.339 \pm 2.5980$ & $0.972(\mathrm{NS})$ & $40 \%$ \\
\hline Karapadasuptata (Numbness In Palm And Foot) & $1.001 \pm 2.013$ & $2.273 \pm 1.9780$ & $0.065(\mathrm{NS})$ & $24 \%$ \\
\hline $\begin{array}{l}\text { Gala Talu Shosha (Dryness In Mouth) } \\
\text { Pindikodveshtana (Cramps) }\end{array}$ & $1.094 \pm 1.002$ & $0.8621 \pm 0.9901$ & $0.620(\mathrm{NS})$ & $21 \%$ \\
\hline Ati Pipasa (Excessive Thirst) & $1.5927 \pm 1.6743$ & $1.54603 \pm 0.8710$ & $0.0003(\mathrm{HS})$ & $67 \%$ \\
\hline Swedadhikya (Excess Perspiration) & $0.9231 \pm 0.8071$ & $0.4462 \pm 0.8023$ & $0.0678(\mathrm{NS})$ & $25 \%$ \\
\hline
\end{tabular}




\section{Intra and Inter Group Comparison of Objective Parameters}

Objective Parameters

Table 3: Effect of Trial Treatment on Fasting Blood Sugar

\begin{tabular}{l|l|l|l|l|l|l|} 
Groups & $\begin{array}{l}\text { Baseline } \\
\text { Mean } \pm \text { SD }\end{array}$ & FU 1 & FU 2 & FU 3 & P Value & $\begin{array}{l}\text { Response } \\
\text { Calculation }\end{array}$ \\
\hline Group A & $196.8 \pm 78$ & $161.5 \pm 45.94$ & $142.7 \pm 31.90$ & $134.9 \pm 25.08$ & $\begin{array}{l}<0.0001 \\
\text { (ANOVA) }\end{array}$ & $31.4 \%$ \\
\hline Group B & $193.8 \pm 71.48$ & $187.1 \pm 69.18$ & $170.1 \pm 61.21$ & $175.25 \pm$ & $\begin{array}{l}0.6308(\mathrm{~ns}) \\
\text { (ANOVA) }\end{array}$ & $9.5 \%$ \\
\hline
\end{tabular}

Between the $\mathrm{P}$ value $=<0.0001$ ( HS) (t test)

group

Fig. 1: Effect of Trial Treatment on Fasting Blood Sugar

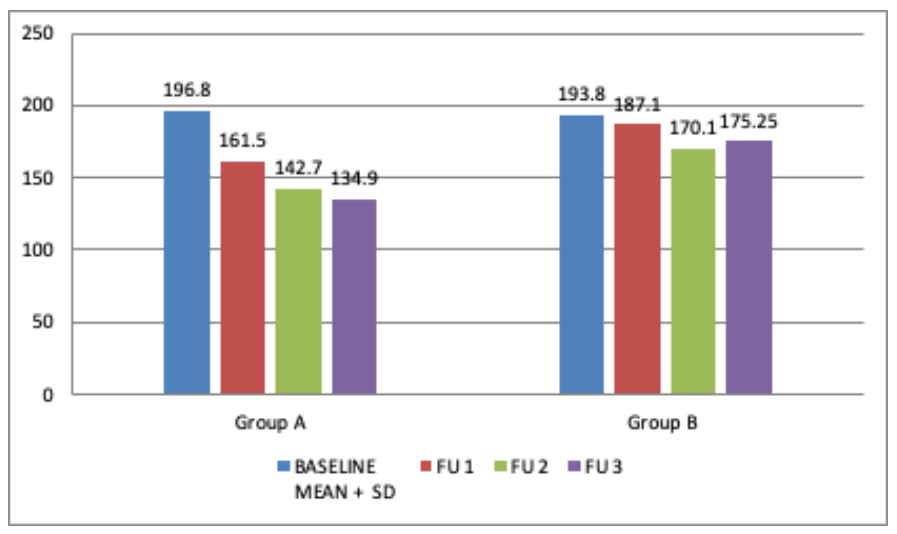

Fig. 2: Post Prandial Blood Sugar

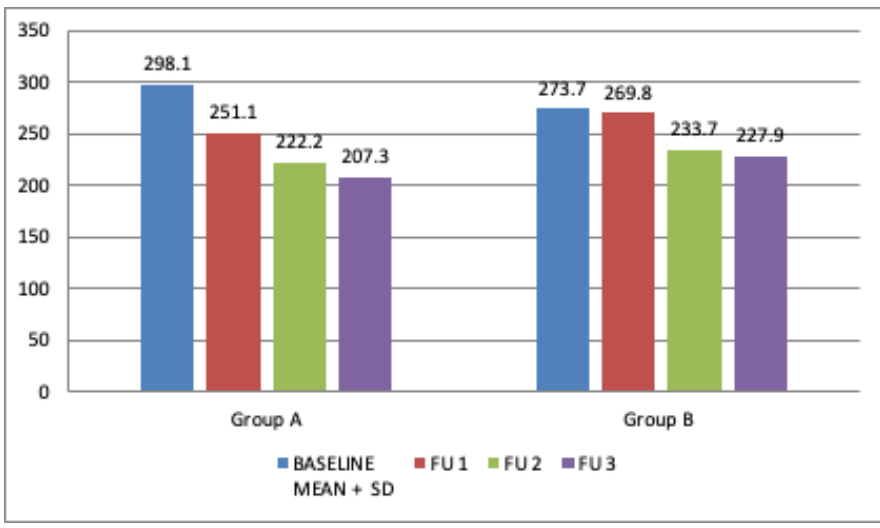

Table 4: Post Prandial Blood Sugar

\begin{tabular}{|l|l|l|l|l|l|r|} 
Groups & $\begin{array}{l}\text { Baseline } \\
\text { Mean } \pm \text { SD }\end{array}$ & FU 1 & FU 2 & FU 3 & P Value & $\begin{array}{l}\text { Response } \\
\text { Calculation }\end{array}$ \\
\hline Group A & $298.1 \pm 76.44$ & $251.1 \pm 76.44$ & $222.2 \pm 49.24$ & $207.3 \pm 56.94$ & $\begin{array}{l}<0.0001(\mathrm{HS}) \\
\text { (ANOVA) }\end{array}$ & $30.4 \%$ \\
\hline Group B & $273.7 \pm 99.19$ & $269.8 \pm 99.95$ & $233.7 \pm 72.31$ & $227.9 \pm 67.71$ & $\begin{array}{l}0.0448(\mathrm{~s}) \\
\text { (ANOVA) }\end{array}$ & $16.7 \%$ \\
\hline
\end{tabular}

Between the $\mathrm{P}$ value $=<0.0001$ ( HS) ( $\mathrm{t}$ test)

group

Table 5: Glycated Hemoglobin $\mathrm{HbA}_{1} \mathrm{C}$

\begin{tabular}{|l|l|l|l|} 
& $\begin{array}{l}\text { Baseline } \\
\text { Mean } \pm \text { SD }\end{array}$ & After Treatment & $\begin{array}{l}\text { P Value } \\
\text { (t test) }\end{array}$ \\
\hline Group A & $9.515 \pm 2.297$ & $8.145 \pm 1.637$ & $<0.0001$ \\
\hline Group B & $8.985 \pm 4.761$ & $8.113 \pm 2.891$ & 0.0029 \\
\hline Between the Group & P value $=0.0382(\mathrm{~S})(\mathrm{t}$ test $)$ & & \\
\hline
\end{tabular}

Fig. 3 : Glycated Haemoglobin $\mathrm{HbA}_{1} \mathrm{C}$

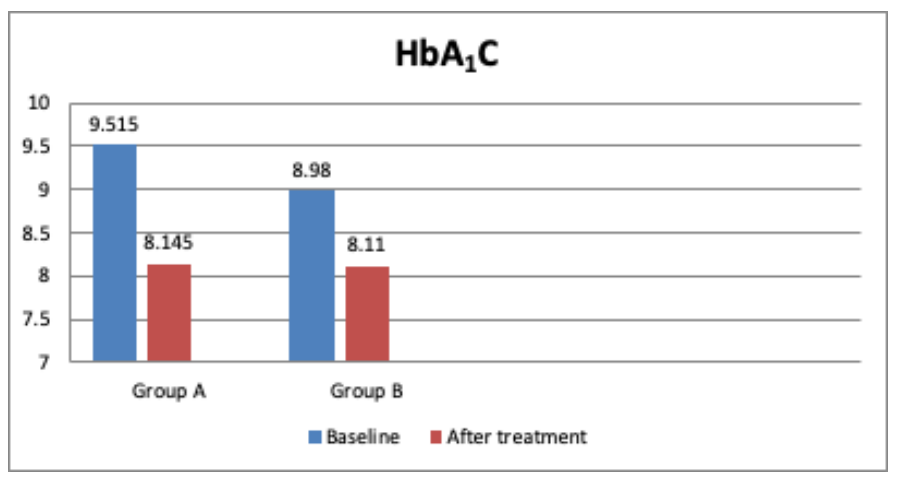

Fig.4: Urine Fasting Glucose

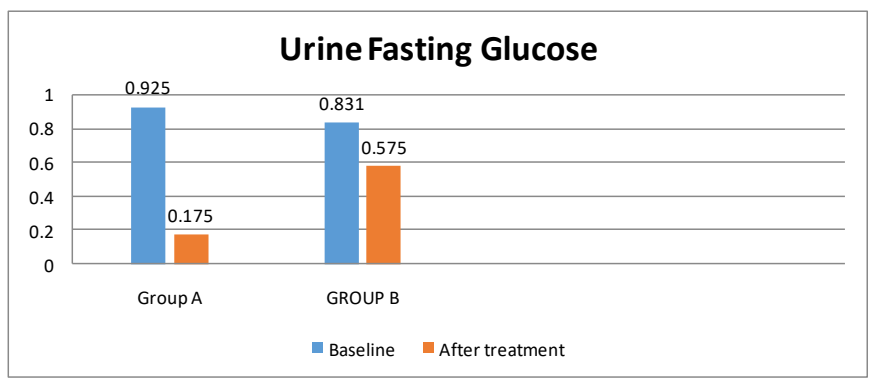


Table 6: Urine Fasting Glucose

\begin{tabular}{|l|l|l|l|} 
& \multicolumn{1}{|c|}{$\begin{array}{c}\text { Baseline } \\
\text { Mean } \pm \text { SD }\end{array}$} & \multicolumn{1}{c|}{ After Treatment } & \multicolumn{1}{c|}{$\begin{array}{c}\text { P Value } \\
(\mathrm{t} \text { test) }\end{array}$} \\
\hline Group A & $0.925 \pm 1.163$ & $0.1750 \pm 0.3848$ & $<0.0001(\mathrm{HS})$ \\
\hline Group B & $0.863 \pm 1.961$ & $0.5750 \pm 0.848$ & $0.0003(\mathrm{Hs})$ \\
\hline Between the group & P value $=0.0371(\mathrm{~S})(\mathrm{t}$ test) & & \\
\end{tabular}

Intra and Inter Group Comparison of Kapha Prakruti Patients

Objective Parameters

Table 7: Fasting Blood Sugar

\begin{tabular}{|l|l|l|l|l|l|}
\multicolumn{1}{c|}{ Groups } & \multicolumn{1}{c}{$\begin{array}{c}\text { Baseline } \\
\text { Mean } \pm \text { SD }\end{array}$} & \multicolumn{1}{c|}{ FU 1 } & \multicolumn{1}{c|}{ FU 2 } & \multicolumn{1}{c|}{ FU 3 } & \multicolumn{1}{c|}{$\begin{array}{c}\text { P Value } \\
\text { (ANOVA) }\end{array}$} \\
\hline Group A & $200.6 \pm 71.41$ & $163.4 \pm 40.09$ & $147.6 \pm 32.50$ & $139.3 \pm 28.18$ & $<0.0001(\mathrm{HS})$ \\
\hline Group B & $199.8 \pm 77.10$ & $194.9 \pm 77.93$ & $184.9 \pm 61.21$ & $174.4 \pm 52.48$ & $0.0171(\mathrm{~S})$ \\
\hline Between the group & P value $=0.0362(\mathrm{~S})(\mathrm{t}$ test) & & & \\
\hline
\end{tabular}

Table 8: Post prandial blood sugar

\begin{tabular}{|l|l|l|l|l|l|}
\multicolumn{1}{c|}{ Groups } & \multicolumn{1}{c|}{$\begin{array}{c}\text { Baseline } \\
\text { Mean } \pm \text { SD }\end{array}$} & \multicolumn{1}{c|}{ FU 1 } & \multicolumn{1}{c|}{ FU 2 } & \multicolumn{1}{c|}{ FU 3 } & $\begin{array}{c}\text { P Value } \\
\text { (ANOVA) }\end{array}$ \\
\hline Group A & $324.8 \pm 111.1$ & $255.0 \pm 75.11$ & $231.8 \pm 48.20$ & $206.5 \pm 57.54$ & $<0.0001(\mathrm{HS})$ \\
\hline Group B & $330.8 \pm 97.40$ & $328.7 \pm 98.90$ & $291.1 \pm 54.44$ & $279.2 \pm 49.04$ & $0.0303(\mathrm{~S})$ \\
\hline Between the group & P value $=0.0632(\mathrm{NS})(\mathrm{t}$ test) & & & \\
\hline
\end{tabular}

Table 9: Glycated haemoglobin $\mathrm{HbA}_{1} \mathrm{C}$

\begin{tabular}{|l|l|l|l|}
\hline \multicolumn{1}{|c|}{ Groups } & \multicolumn{1}{c|}{$\begin{array}{c}\text { Baseline } \\
\text { Mean } \pm \text { SD }\end{array}$} & \multicolumn{1}{c|}{ After Treatment } & \multicolumn{1}{c|}{$\begin{array}{c}\text { P Value } \\
\text { (t test) }\end{array}$} \\
\hline Group A & $10.19 \pm 8.66$ & $8.66 \pm 1.76$ & 0.0005 \\
\hline Group B & $9.348 \pm 6.61$ & $8.113 \pm 2.891$ & 0.0369 \\
\hline Between the Group & P value $=0.045(\mathrm{~S})(\mathrm{t}$ test) & & \\
\hline
\end{tabular}

Intra and Inter Group Comparison of Vata Prakruti Patients

Objective Parameters

Table 10: Fasting Blood Sugar

\begin{tabular}{|l|c|c|c|c|c|}
\multicolumn{1}{c|}{ Groups } & $\begin{array}{c}\text { Baseline } \\
\text { Mean } \pm \text { SD }\end{array}$ & \multicolumn{1}{c|}{ FU 1 } & FU 2 & FU 3 & $\begin{array}{c}\text { P Value } \\
\text { (ANOVA) }\end{array}$ \\
\hline Group A & $182.1 \pm 87.25$ & $158.7 \pm 54.85$ & $135.2 \pm 29.07$ & $127.7 \pm 18.18$ & $0.0019(\mathrm{~S})$ \\
\hline Group B & $155.2 \pm 71.41$ & $148.7 \pm 40.09$ & $147.1 \pm 32.50$ & $143.1 \pm 40.68$ & $0.0378(\mathrm{~S})$ \\
\hline Between the group & P value $=0.0362(\mathrm{~S})(\mathrm{t}$ test) & & & \\
\hline
\end{tabular}

Table 11: Post prandial blood sugar

\begin{tabular}{|l|l|l|l|l|l|}
\multicolumn{1}{c|}{ Groups } & \multicolumn{1}{c|}{$\begin{array}{c}\text { Baseline } \\
\text { Mean } \pm \text { SD }\end{array}$} & \multicolumn{1}{c|}{ FU 1 } & \multicolumn{1}{c|}{ FU 2 } & \multicolumn{1}{c|}{ FU 3 } & \multicolumn{1}{c|}{$\begin{array}{c}\text { P Value } \\
\text { (ANOVA) }\end{array}$} \\
\hline Group A & $267.2 \pm 95.1$ & $244.4 \pm 84.52$ & $204.2 \pm 52.90$ & $211.4 \pm 60.37$ & $0.0053(\mathrm{~S})$ \\
\hline Group B & $232.7 \pm 76.80$ & $226.8 \pm 76.40$ & $195.8 \pm 60.36$ & $193.7 \pm 58.84$ & $<0.0001(\mathrm{HS})$ \\
\hline Between the group & P value $=0.094(\mathrm{~N} \mathrm{~S})(\mathrm{t}$ test) & & & \\
\hline
\end{tabular}

Table 12: Glycated haemoglobin $\mathrm{HbA}_{1} \mathrm{C}$

\begin{tabular}{|l|l|l|l|} 
& \multicolumn{1}{|c|}{$\begin{array}{c}\text { Baseline } \\
\text { Mean } \pm \text { SD }\end{array}$} & \multicolumn{1}{c|}{ After Treatment } & $\begin{array}{c}\text { P Value } \\
\text { (t test) }\end{array}$ \\
\hline Group A & $9.09 \pm 7.14$ & $8.98 \pm 5.76$ & $0.0293(\mathrm{NS})$ \\
\hline Group B & $9.14 \pm 8.51$ & $9.01 \pm 6.88$ & $0.692(\mathrm{~S})$ \\
\hline Between the Group & P value $=0.911(\mathrm{NS})(\mathrm{t}$ test) & &
\end{tabular}

\section{Intra and Inter Group Comparison of Pitta Prakruti Patients Objective Parameters}

Table 13: Fasting Blood Sugar

\section{Groups}

Group A

Group B

Between the group
Baseline

Mean \pm SD

$200.6 \pm 71.41$

$199.8 \pm 77.10$

P value $=0$
FU 1

FU 2

$163.4 \pm 40.09$

$194.9 \pm 77.93$

NS)( $($ test $)$

\section{FU 3}

$183.3 \pm 28.18$

$174.4 \pm 52.48$
PValue

(ANOVA)

0.932(NS)

$0.0201(\mathrm{~S})$ 


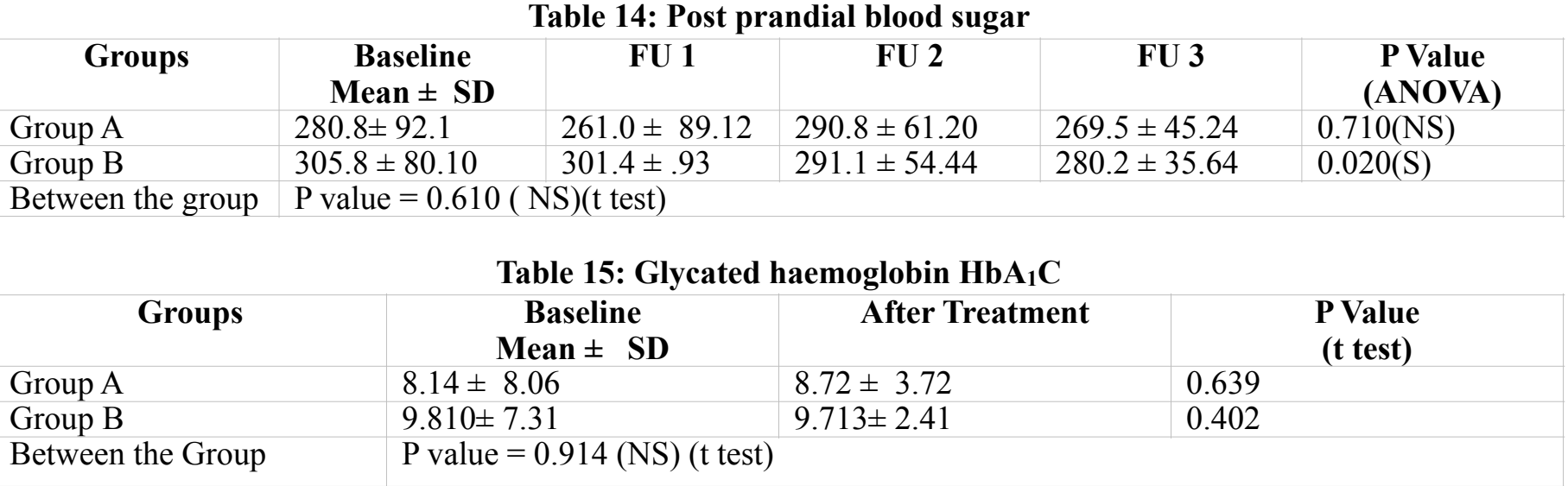

\section{SUBJECTIVE PARAMETERS}

Intra and Inter Group Comparison of Kapha Prakruti Patients

Table 16: Effect of Trial Treatment on Polyuria (Prabhutamutrata)

\begin{tabular}{|c|c|c|c|c|}
\hline Groups & $\begin{array}{r}\text { Prabhut } \\
\text { MEA }\end{array}$ & $\begin{array}{l}\text { trata } \\
\text { DD }\end{array}$ & $\begin{array}{l}\text { P Value } \\
\text { (t test) }\end{array}$ & $\begin{array}{l}\text { Percentage of } \\
\text { relief }\end{array}$ \\
\hline & BT & AT & & \\
\hline A & $3.214 \pm 1.019$ & $0.291 \pm 0.671$ & $<0.0001$ & $90 \%$ \\
\hline $\mathrm{B}$ & $2.902 \pm 1.193$ & $1.820 \pm 0.719$ & 0.437 & $36 \%$ \\
\hline en the group & $\mathrm{P}$ value $=<0.0001(\mathrm{t}$ test $)$ & & HS & \\
\hline
\end{tabular}

Table 17: Effect of Trial Treatment on Laziness (Alasya)

\begin{tabular}{|c|c|c|c|c|}
\hline \multirow[t]{2}{*}{ Groups } & \multicolumn{2}{|c|}{$\begin{array}{c}\text { Alasya } \\
\text { MEAN } \pm \text { SD }\end{array}$} & \multirow[t]{2}{*}{$\begin{array}{c}\text { P VALUE } \\
\text { (t test) }\end{array}$} & \multirow[t]{2}{*}{$\begin{array}{l}\text { Percentage } \\
\text { of relief }\end{array}$} \\
\hline & BT & AT & & \\
\hline Group A & $3.001 \pm 1.934$ & $0.491 \pm 0.602$ & $<0.0001$ & $83 \%$ \\
\hline Group B & $2.313 \pm 1.213$ & $1.261 \pm 0.861$ & 0.0017 & $45 \%$ \\
\hline $\begin{array}{l}\text { Between the group } \\
\text { comparison }\end{array}$ & $\mathrm{P}$ value $=<0.0001(\mathrm{t}$ test $)$ & $\mathrm{HS}$ & & \\
\hline
\end{tabular}

Table 18: Effect of Trial Treatment on Excessive Eating (Atikshudha)

\section{Groups}

Group A

Group B

Between the group

comparison

Atikshudha
MEAN \pm SD

$2.905 \pm 1.810$

$2.973 \pm 1.213$

$\mathrm{P}$ value $=<0.0001(\mathrm{t}$ test $)$
P VALUE

(t test)

\section{Percentage \\ of relief}

\begin{tabular}{|l|l|l|l|}
\hline BT & \multicolumn{1}{|c|}{ AT } & \multicolumn{1}{|c}{ (t test) } & \multicolumn{1}{c}{ of re } \\
& $0.491 \pm 0.602$ & $<0.0001$ & $83.1 \%$ \\
\hline & $1.261 \pm 0.861$ & 0.0032 & $56 \%$ \\
\hline
\end{tabular}

\section{INTRA AND INTER SUB GROUP COMPARISION OF VATA PRAKRITI PATIENTS}

Table 19: Effect of Trial Treatment on Prabhutamutrata (Polyuria)

\section{Groups}

\begin{tabular}{|l|}
\hline \multicolumn{1}{|c|}{ Groups } \\
\hline Group A \\
\hline Group B \\
\hline $\begin{array}{l}\text { Between the group } \\
\text { comparison }\end{array}$ \\
\hline
\end{tabular}

Prabhuta Mutrata

MEAN \pm SD

BT

$$
3.924 \pm 1.18
$$

$2.810 \pm 0.713$

$\mathrm{P}$ value $=<0.0001(\mathrm{t}$ test $)$
P VALUE

(t test)

\section{Percentage} of relief

Table 20: Effect of Trial Treatment on Daurbalya (Weakness)

\section{Groups}

Group A

Group B

Between the group

comparison

\section{Daurbalya}

MEAN \pm SD

BT

$2.905 \pm 2.18$

$2.134 \pm 1.17$

$\mathrm{P}$ value $=<0.0001(\mathrm{t}$ test $)$
$0.183 \pm 0.934$

$1.634 \pm 0.451$
$<0.0001$

0.078

HS
$90 \%$ $37 \%$

(1)

\begin{tabular}{|c|c|c|c|}
\hline \multicolumn{2}{|c|}{$\begin{array}{c}\text { Daurbalya } \\
\text { MEAN } \pm \text { SD }\end{array}$} & \multirow[t]{2}{*}{$\begin{array}{l}\text { P VALUE } \\
\text { (t test) }\end{array}$} & \multirow[t]{2}{*}{$\begin{array}{l}\text { Percentage } \\
\text { of relief }\end{array}$} \\
\hline BT & $\mathbf{A T}$ & & \\
\hline & $0.870 \pm 0.918$ & $<0.0001$ & $69 \%$ \\
\hline & $1.851 \pm 0.621$ & 0.0401 & $13 \%$ \\
\hline $1(\mathrm{t} t \mathrm{~s}$ & & HS & \\
\hline
\end{tabular}


Intra and Inter Sub Group Comparison of Pittaprakruti Patients

Table 21: Effect of Trial Treatment on Swedadhikya (Excessive Perspiration)

\begin{tabular}{|c|c|c|c|c|}
\hline \multirow[t]{2}{*}{ Groups } & \multicolumn{2}{|c|}{$\begin{array}{l}\text { Swedadhikya } \\
\text { MEAN } \pm \text { SD }\end{array}$} & \multirow[t]{2}{*}{$\begin{array}{l}\text { P VALUE } \\
\text { (t test) }\end{array}$} & \multirow[t]{2}{*}{$\begin{array}{l}\text { Percentage } \\
\text { of relief }\end{array}$} \\
\hline & BT & AT & & \\
\hline Group A & $1.981 \pm 1.206$ & $0.195 \pm 0.721$ & $<0.0295$ & $90.4 \%$ \\
\hline Group B & $2.171 \pm 1.920$ & $1.973 \pm 0.416$ & 0.437 & $9 \%$ \\
\hline $\begin{array}{l}\text { Between the group } \\
\text { comparison }\end{array}$ & $\mathrm{P}$ value $=<0.0001(\mathrm{t}$ test $)$ & & HS & \\
\hline
\end{tabular}

Table 22: Effect of Trial Treatment on Daurbalya (Weakness)

\begin{tabular}{|c|c|c|c|c|}
\hline \multirow[t]{2}{*}{ Groups } & \multicolumn{2}{|c|}{$\begin{array}{c}\text { Daurbalya } \\
\text { MEAN } \pm \text { SD }\end{array}$} & \multirow[t]{2}{*}{$\begin{array}{l}\text { P VALUE } \\
\text { (t test) }\end{array}$} & \multirow[t]{2}{*}{$\begin{array}{l}\text { Percentage } \\
\text { of relief }\end{array}$} \\
\hline & BT & AT & & \\
\hline Group A & $2.193 \pm 2.02$ & $0.951 \pm 0.932$ & $<0.0001$ & $56 \%$ \\
\hline Group B & $2.296 \pm 1.831$ & $0.193 \pm 0.821$ & 0.0003 & $91.4 \%$ \\
\hline $\begin{array}{l}\text { Between the group } \\
\text { comparison }\end{array}$ & $\mathrm{P}$ value $=<0.0001(\mathrm{t}$ test $)$ & & HS & \\
\hline
\end{tabular}

Table23: Effect of Trial Treatment on Karpadadaha (burning sensation in hand and feet)

\begin{tabular}{|c|c|c|c|c|}
\hline \multirow[t]{2}{*}{ Groups } & \multicolumn{2}{|c|}{$\begin{array}{l}\text { Karpadadaha } \\
\text { MEAN } \pm \text { SD }\end{array}$} & \multirow[t]{2}{*}{$\begin{array}{l}\text { P VALUE } \\
\text { (t test) }\end{array}$} & \multirow[t]{2}{*}{$\begin{array}{c}\text { Percentage } \\
\text { of relief }\end{array}$} \\
\hline & BT & AT & & \\
\hline A & $2.182 \pm 1.962$ & $1.061 \pm 0.692$ & $<0.004$ & $47 \%$ \\
\hline B & $2.567 \pm 1.819$ & $2.791 \pm 0.813$ & 0.921 & - \\
\hline $\begin{array}{l}\text { een the group } \\
\text { arison }\end{array}$ & $P$ value $=<0.0001(\mathrm{t}$ test $)$ & & HS & \\
\hline
\end{tabular}

Inter subgroup comparison of Vata and Kapha HerbalTea in Group A

Table 24: Fasting blood glucose

\begin{tabular}{|l|c|c|c|c|c|}
\hline \multicolumn{1}{|c|}{ Groups } & \multicolumn{1}{|c|}{$\begin{array}{c}\text { Baseline } \\
\text { Mean } \pm \text { SD }\end{array}$} & \multicolumn{1}{c|}{ FU 1 } & FU 2 & FU 3 & \multicolumn{1}{c|}{ P Value } \\
\hline $\begin{array}{l}\text { Group A Vata } \\
\text { Prakriti tea }\end{array}$ & $182.1 \pm 87.25$ & $158.7 \pm 54.85$ & $135.2 \pm 29.07$ & $127.7 \pm 18.18$ & $\begin{array}{l}0.0019(\mathrm{~S}) \\
\text { (ANOVA) }\end{array}$ \\
\hline $\begin{array}{l}\text { Group A Kapha } \\
\text { Prakriti tea }\end{array}$ & $200.6 \pm 71.41$ & $163.4 \pm 40.09$ & $147.6 \pm 32.50$ & $139.3 \pm 28.18$ & $<0.0001(\mathrm{HS})$ \\
$\begin{array}{l}\text { Betwe en the } \\
\text { Prakriti }\end{array}$ & P value $=0.572$ (NS) (t test) & & & \\
\hline
\end{tabular}

Table 25: Post prandial blood sugar

\begin{tabular}{|c|c|c|c|c|c|}
\hline Groups & $\begin{array}{c}\text { Baseline } \\
\text { Mean }+ \text { SD }\end{array}$ & FU 1 & FU 2 & FU 3 & P Value \\
\hline Group A Vata Prakriti tea & $267.2 \pm 95.1$ & $244.4 \pm 84.52$ & $204.2 \pm 52.90$ & $211.4 \pm 60.37$ & $\begin{array}{l}0.0053(\mathrm{~S}) \\
\text { (ANOVA) }\end{array}$ \\
\hline $\begin{array}{l}\text { Group A KaphaPrakriti } \\
\text { tea }\end{array}$ & $324.8 \pm 111.1$ & $255.0 \pm 75.11$ & $231.8 \pm 48.20$ & $206.5 \pm 57.54$ & $\begin{array}{l}<0.0001 \text { (HS) } \\
\text { (ANOVA) }\end{array}$ \\
\hline
\end{tabular}

Table 26: Glycated haemoglobin A1c

\begin{tabular}{l|l|l|l|}
\multicolumn{1}{c|}{ Groups } & \multicolumn{1}{c|}{$\begin{array}{c}\text { Baseline } \\
\text { Mean } \pm \text { SD }\end{array}$} & \multicolumn{1}{c|}{ After Treatment } & \multicolumn{1}{c}{$\begin{array}{c}\text { P Value } \\
\text { (t test })\end{array}$} \\
\hline Group A Vata Prakriti tea & $9.09 \pm 7.14$ & $8.98 \pm 5.76$ & 0.0293 \\
\hline $\begin{array}{l}\text { Group A KaphaPrakriti } \\
\text { tea }\end{array}$ & $9.348 \pm 6.61$ & $8.113 \pm 2.891$ & 0.0369 \\
\hline $\begin{array}{l}\text { Between the Group } \\
\text { P value }=0.721 \text { ( NS) (t test) }\end{array}$ & &
\end{tabular}


Groups

Table 27: Prabhutamutrata (Polyuria)

\begin{tabular}{|l|l|l|l|l|}
\hline \multirow{2}{*}{ Groups } & \multicolumn{2}{|c|}{$\begin{array}{c}\text { Prabhuta Mutrata } \\
\text { Mean } \pm \text { SD }\end{array}$} & \multicolumn{1}{|c|}{$\begin{array}{c}\text { P Value } \\
\text { (t test) }\end{array}$} & $\begin{array}{c}\text { Percentage } \\
\text { of relief }\end{array}$ \\
\cline { 2 - 3 } & \multicolumn{1}{|c|}{ BT } & \multicolumn{1}{c|}{ AT } & & \\
Group A Vata Prakriti tea & $3.214 \pm 1.019$ & $0.291 \pm 0.671$ & $<0.0001$ & $90 \%$ \\
\hline Group A Kapha Prakriti tea & $3.924 \pm 1.18$ & $0.183 \pm 0.934$ & $<0.0001$ & $94 \%$ \\
\hline Between the group comparison & P value $=<0.0001(\mathrm{t}$ test) & HS & &
\end{tabular}

\section{Discussion}

The Prakriti approach is adopted for assessment of proneness of the disease (7), diagnosis and treatment to incorporate appropriate diet plan and regimen.In the present study $45.3 \%$ Kapha Pradhana prakriti (KaphaVatapradhana $=28 \%$, Kaphapittapradhana prakriti $=17.3 \%$ ) patients were found. This shows that Diabetes Mellitus correlated to Prameha Kaphapradha vyadhi is prevelant in Kapha prakriti individuals. This is similar to study conducted in SDM Hassan, in which 83 \% Kapha Pradhana prakriti (KaphaVatapradhana $=48 \%$, Kaphapittapradhana prakriti $=35 \%$ ) patients were found (7). If proper Kaphahara diet and lifestyle is implemented since childhood properly, incidence can be reduced to marked extent.

In the present study the intragroup comparison findings between Herbal Tea group and control group were found highly significant in Fasting, (fig 1) Post Prandial Blood Glucose (fig 2) and $\mathrm{HbA}_{1} \mathrm{C}$ (fig 3). Reduction in Urine Glucose showed significant results with $\mathrm{p}$ value 0.0371 . (fig 4). Serum Cholesterol and Serum Triglyceride values were found nonsignificant as the values were within normal range.

\section{Role of Kapha Herbal Tea in Kaphapradhana Prakriti Diabetes Patients}

Kapha has Madhura (Sweet), Sheeta (Cold), Sthira (Stable), Pichilla (slimmy), Guru (Heavy) properties, the ingredients used in Kapha Herbal Tea were Katu (pungent), Tikta (bitter), Kashya (astringent) Rasa Pradhana, Laghu (light), Ushna (hot), Kapha or Tridoshashamak and Mehahara (antidiabetic) properties. Research studies on Sunthi (Zingiber officinale Roxb) shows that it is a potent treatment for Diabetes Mellitus. The intra and inter group comparison of Kaphaja Herbal Tea on fasting blood glucose was highly significant with $\mathrm{p}$ value $<0.0001$ and 0.0362 respectively (Table7). This effect seen may be contributed to the effect of ginger as seen in previous study which shows $24-53 \%$ reduction of fasting blood Glucose (8). Twak (9) and Jambu both have significant effect on fasting glucose levels seen both in animal studies and Randomized controlled trials, one of the study observed a statistically significant reduction $(\mathrm{P}<$ 0.001 ) by $10.6 \%$ in the mean fasting blood glucose levels (10). Clove (Syzygium aromaticum Linn.) flower buds extract significantly reduce the blood glucose level in Type 2 Diabetic KK-A(y) mice (11) Similarly the intra group comparison of Kaphaja Herbal Tea in group A on post prandial blood sugar was highly significant with $p$ value $<0.0001$.(Table 8 ). This reduction in post prandial Blood Sugar levels probably seen with the effect of Meshashringi (Gymnema sylvestre). The mode of action of the drug is through stimulation in insulin secretion from pancreas(12) Stem bark of Ceylon cinnamon (Twak ) Cinnamtannin B1, activates the phosphorylation of the insulin receptor $\beta$-subunit on adipocytes as well as other insulin receptors (13). Further the action of herbs like Jambu (14), Lavanga (15), Tulasi (16) on PPBS is seen in various studies with potent reduction of $17.6 \%$ and $7.3 \%$ in the levels of fasting and postprandial blood glucose, respectively. Activities of Myristica fragrans Houtt nutmeg have also reported to its insulin-like biological activity (17) The effect of the Gymnema sylvestre leaf extracts are highly beneficial for subjects suffering from Diabetes mellitus and is synonymous to 4 unit $/ \mathrm{kg}$ of Insulin (18) Piperine for 2 weeks partially protects against Diabetes induced oxidative stress (19) The aqueous extract of leaves of Ocimum sanctum Linn. showed the significant reduction in Blood Sugar level in both normal and Alloxan induced diabetic $\operatorname{rat}(20)$. The values of $\mathrm{HbA}_{1} \mathrm{C}$ were highly significant on intra group comparison of Kaphaja Herbal Tea in group A (Table 9). The reduction of Glycated Haemoglobin level is found probably due to effect of cinnamon which demonstrated $\mathrm{HbA}_{1} \mathrm{C}$ reduction of $40.2 \%$ in cinnamon treated rats compared to untreated Diabetic Rats(21)Cinnamon (Cinnamomum zeylanicum Breyn) is dietary component that has been shown to contain biologically active substances that have insulin-mimetic properties and regulate blood glucose, it enhances glucose uptake by activating insulin receptor kinase activity, auto-phosphorylation of the insulin receptor and glycogen synthase activity. The cinnamon extracts shown better Glycaemic control in Diabetes-induced animals as demonstrated by the stable HbA1c in the cinnamon group as opposed to the Diabetic control group (22). However results were nonsignificant in inter group comparisons.

\section{Role of Vata Herbal Tea in Vatapradhana Prakriti Diabetes Patients}

The second highest prevalent Prakriti is seen in the present study was Vata Pradhana prakriti which is $44 \%$ (VataKaphapradhana $=24 \%$, Vatapittapradhana prakriti $=20 \%)$. This shows the importance of vata in Diabetes Mellitus which is Kapha Vata Anubandh Tridoshja vyadhi and as it is said that Madhumeha is the last subtype of Vataja Prameha. Vata due to its Ruksha (dry), Laghu (light) Guna deteriorate the Dhatus which causes either Avarana or Kshaya of Dhatus leading to Madhumeha which becomes incurable due to lots of complications.Hence balancing of vata is equally important in management of disease. 
The ingredients used in Vata Herbal Tea having Vatapradhana doshashamaka or Tridoshashamaka properties. Few Ingredients of Vata Herbal Tea are same as in Kapha Herbal tea due to their same Doshashamaka action like Sunthi, Meshashringi, Jatiphala, Lavanga, Tulasi, these all are potent antidiabetic herbs as discussed earlier. Further, other ingredients. A single study by Barros et al (23) reported that fennel can improve rat glucose tolerance.Similarly the Tagar (Valeriana wallichii) plant also possesses Anti-Diabetic properties thus can be used for treating individuals suffering from Diabetes (24). A recent systematic review and meta-analysis of valerian evidenced, that valerian is a safe herb associated with only rare adverse events (25) Another spice is Tejapatra (C. tamala), extract of $C$. tamala exhibit significant antihyperglycemic activities in STZ-induced rats. The extract also showed improvement in lipid profile, body weight and oral glucose tolerance test (OGTT) results, hence might be valuable in Diabetes (26).The intra group comparison of Vata Herbal Tea in Group A on Fasting Blood sugar was highly significant with $p$ value $<0.0001$ and maximum difference in mean values were observed (Table 10). The observed results in Group A is due to the antidiabetic effect of Tulasi , Sunthi, Lavanga ,Tagar as discussed earlier. The effect of the Gymnema sylvestre leaf extracts is synonymous to $4 \mathrm{unit} / \mathrm{kg}$ of insulin thus highly beneficial for individuals suffering from Diabetes Mellitus (27), The leafs of Cinnamomum tamala leaves showed significant results in diabetic rats (28) further the extract of Cuminum cyminum possesses anti-diabetic effect in Diabetic Rats through reduction of Plasma Glucose levels and elevation of insulin in plasma (29). The results on postprandial blood sugar were found significant in Herbal Tea group (Table 11) which is seen due to effective Anti hyperglycemic effect of Ela (Elaterria cardomum Maton) as supplementation by suppression of $\alpha$-amylase and $\alpha$-glucosidase enzymes may regulate glucose metabolism (30) in addition to the other antidiabetic herbs used in Vata tea as mentioned earlier. Similarly the intra group comparison of $\mathrm{HBA}_{1} \mathrm{C}$ in Vata Herbal Tea in group A was significant (Table 12). This antidiabetic effect may be achieved by cumin seeds which has marked results on $\mathrm{HbA}_{1} \mathrm{C}$ reduction along with lipid profile (31) levels. Along with it another herb Myrstica fragnansHoutt evidenced significant reduction of glucose and triglyceride (TG) levels (maximal effect of 53\% reduction of glucose) (32).

\section{Role of Pitta Herbal Tea in Pittapradhana Prakriti Diabetes Patients}

Pitta is the most important factor responsible for Samagni. Metabolism is regulated by pitta only. One study describes the concept of Prakriti in aging stating that the pitta predominance Prakriti type individuals have high basal metabolic rate (BMR) and energy consumption leading to tissue destruction and premature aging and average life span, while Kapha predominance Prakriti type have a tendency to delayed manifestation of aging and longer life $\operatorname{span}(33)$.
Diabetes is a metabolic disease which is initiated by Agnimandya leading to Ama formation that result in Apachita Dhatus and finally the disease. As stated earlier in this study while assessing the Prakriti of the subjects enrolled Pittaanubandha is seen in 50 percent of the cases, although Pitta Pradhana Prakriti subjects were less, Pittakaphaja - 4\% and Pitta Vataja - 6\%.

The ingredients of Pitta Herbal Tea were not very Ushna, Tikshna to balances the Prakritidosha, but the ingredients are mainly Agni Deepana, Pachana and Tikta Kashaya Rasa Pradhana. The Herbs have Pittahara, Tridoshara Properties. The major ingredient of this tea is Asana (Pterocarpus marsupium Roxb.) is called Rasayana (34). In Ayurveda, aqueous extract of heart-wood of $P$. marsupium is used in treatment of Diabetes (35) Although there are several reports on $P$. marsupium as Anti-Diabetic (36)(37)(38).

According to Acharyas, Nishaamalaki is described as a potent treatment for Diabetes mellitus. The spice Turmeric, which is derived from the root of the plant Curcuma longa Linn. Administration of various dosages of curcumin in rat models were able to prevent body weight loss, reduce the levels of Glucose, Haemoglobin, and Glycosylated Haemoglobin (HbA1c) in blood (39), and improve insulin sensitivity(40) (41). Another study on coriander leaf and stem extract showed a significant reduce in the blood glucose levels and blood lipids as the total cholesterol, TC, VLDL, LDL Kar et al.(42), has reported that ethanol extract of Ficus racemosa $(250 \mathrm{mg} / \mathrm{kg} / \mathrm{day}$, once, twice, and thrice daily, per oral normalized the blood glucose, lowered the urine sugar, and helped it to reach a level of zero within two weeks, in alloxaninduced diabetic albino Wister rats(43).

In the present study the results of Fasting, (Table 13). Postprandial Blood Glucose (Table 14). and HbAlc(Table 15) found non-significant due to very less sample of Pitta Pradhana prakriti.

\section{Role of Prakriti specific Herbal Tea on Subjective Parameters \\ Kapha Herbal Tea}

In present study there is highly significant improvement in Prabhutamutrata (Table 16) and Alasya(Table 17) and Atikshudha(Table 18)in group A. This result on Prabhutamutrata is propably seen due to Kashaya rasa and Sangrahi gunaofJambu (Syzyzium cumini Linn.)and Tikta rasa of Bilwa Patra along with Mehahara properties of Meshashringi. Laghu (light ), Ruksha (dry) , Ushna (hot ) and Kapha Shamak properties of Brihatela, Lavanga, Twak, Sunthi And Tulasi may acted upon the Alasya guna which is due to increase Guruta and Kapha in body. Sunthi and Kali Mirch, which are Pachana Dravyas may influence the metabolism and hence due to correction of Agni patients feels Kshudha on proper time with normal frequency. Kashaya Tikta Rasa of ingredients cause early satiety hence could prevent overeating.

\section{Vata Herbal Tea}

This study reveals that there is significant improvement in Prabhutamutrata (Table 19) due to 
Kashaya and Tikta Rasa of major ingredients like Jeeraka, Patra, causes reduction in urine quantity and frequency. The ingredients like Sunthi which is Vrishya by Prabhava, Tulasi has immune booster properties. Further herbs like Ela (44) Jeeraka(45) enhances antioxidants in body causing diminishing of symptoms like Daubalya (Table 20).

\section{Pitta Herbal Tea}

In subjective parameters there is improvement in Swedadhikya (Table 21)., Karpadaha,(Table 22) and Daurbalya (Table 23)in Group A. The activity of Asana and Haridra which are Katu, Kashaya may reduce perspiration .The burning sensation of hands and feet may be reduced due to Shheeta properties of Mishreyak, Asana, Udumbara, and Ela. In addition, Antioxidant effect of Haridra, Tulasi, Asana and Ela, Mishreya provide significant result in Daurbalya.

\section{Inter sub group comparison of Vata and Kapha Herbal Tea in Group A (Objective Parameters)}

The inter sub group comparison of Fasting (Table 24), Post prandial Blood Sugar(Table 25) and $\mathrm{HbA}_{1} \mathrm{C}$ (Table 26) levels between Kapha and Vata Herbal Tea shows insignificant results with $\mathrm{p} 0.572$, p 0.078 and $p 0.721$ respectively. This indicates that both the Herbal Teas are equally effective in management of Diabetes Mellitus, Further it is important to analyse the Prakriti before the treatment of any disease which infer the importance of Prakriti specific Herbal Tea in the management of Diabetes mellitus.

Intersubgroup comparison between Kapha and Pitta or Vata and Pitta could not be calculated due to very small sample of Piita Prakriti patients.

\section{Inter sub group comparison of Vata and Kapha Herbal Tea in Group A (Subjective Parameters)}

Prabhuta mutrata was a common symptom which show highly significant results (Table 27) in both the Tea which infer equal effect of both the herbal tea in Prabhuta mutrata

\section{Conclusion}

Prameha can be correlated with diabetes mellitus, with its increased occurrence in Kapha Pradhana Prakriti followed by Vata Pradhana Prakriti. Hence, preventive measures should be adopted from early life among Kapha and Vata pradhana Prakriti individuals. Inter subgroup comparison showed, significant improvements in both objective and subjective parameters among all three Herbal teas. The Kapha Herbal tea has higher efficacy followed by Vata Herbal tea. Pitta Herbal tea the results were statistically not significant, however symptomatic relief was observed in most of the cases. These differences may be due to small sample size. Significant reduction in Fasting Blood sugar, Post prandial Blood Sugar, $\mathrm{HbA1C}$, Urine fasting glucose was observed in Group A (Trial) as compared to Group B (Control). Prakriti specific herbal tea is simple formulation with no reported adverse effects and cost effective. Hence, tea can be substituted with Herbal tea for better management and control of disease.

\section{References}

1. Bommer C, Global Economic Burden of Diabetes in Adults: Projections From 2015 to 2030.,Diabetes Care. 2018 May; 41(5):963-970.

2. Charaka Samhita by Agnivesha with 'Vidyotini Hindi Commentary by Pt. Kashinath Shastry and Dr. Gorakhnath Chaturvedi, Part 1, Chaukhamba Bhauati Academy, Varanasi, reprint 2011, page 630

3. Charaka Samhita with 'Vidyotini Hindi Commentary by Pt. Kashinath Shastry and Dr. Gorakhnath Chaturvedi, Part 2, Chaukhamba Bhauati Academy, Varanasi, reprint 2011, Page 233

4. Akshay A. Agarwal, Pradeep R. Jadhav, and Yeshwant A. Deshmukh, Prescribing pattern and efficacy of anti-diabetic drugs in maintaining optimal glycemic levels in diabetic patients , J Basic Clin Pharm. 2014; 5(3): 79-83.

5. Rastogi Sajneev, Development and validation of a Prototype Prakriti Analysis Tool (PP AT):Inferences from a pilot study, AYU journal,2012, 33(2): 209-218

6. Clinical Evaluation Of Varadi Kwatha In The Management Of Madhumeha (Type-2 Diabetes Mellitus) - An Attempt To Provide Evidence Based Data To The Classical Therapeutic Claims, Gyaneshwarsing Guddoye, 1 B.K. Dwibedy2 And O.P. Singh3, J Res Educ Indian Med, Jan.-March 2014; Vol. Xx (1): 37-44

7. Pdt. Harisadashivshashtri paradkar, Astanga Hridaya SarvangaSundara vyakhya and ayurveda rasayana Tika, Varanasi: Chaukhambha Surbharati Prakashan;2016: Sutra 12/67-68.

8. Annie $M$ sithara et al, a cross sectional survey to analyse Deha prakruti and the major risk factors of type 2 Diabetes Mellitus, international journal research Ayurveda pharmacy , 2015; 6/6: 714-719

9. Minpai kiloda et al ,Hypoglycaemic effects of clove (Syzygium aromaticum flower buds) on genetically diabetic KK-A y mice and identification of the active ingredients, Journal of Natural Medicines 2012, 66 (2): 394-9

10. UK Prospective Diabetes Study (UKPDS) Group. Intensive blood-glucose control with sulphonylureas or insulin compared with conventional treatment and risk of complications in patients with type 2 diabetes (UKPDS 33) Lancet. 1998;352: 837-53

11. Leigh Broadhurst C, Marilyn M. Polansky, Richard A. Anderson. Insulin like Biological Activity of Culinary and Medicinal Plant Aqueous Extracts in vitro. J. Agric. Food Chem. 2000; 48:849-852.

12. Tiwari P, Ahmad K and Hassan Baig M. (2017). Gymnema sylvestre for diabetes: from traditional herb to future's therapeutic. Current pharmaceutical design, 23(11), 1667-76.

13. Krishnapura Srinivasan, Black Pepper (Piper nigrum) and Its Bioactive Compound, Piperine, 
Molecular Targets and Therapeutic Uses of Spices, May 2009:25-64.

14. Vats V., Grover J.K., Ra,thi S.S. Evaluation of antihyperglycaemic and hypoglycaemic effect of Trigonella foenum-graecum Linn, Ocimum sanctum Linn and Pterocarpus marsupium Linn in normal and alloxanized diabetic rats. J. Ethnopharmacol. 2002; 79:95-100

15. Ojewole JAO. Analgesic, anti-inflammatory and hypoglycaemic effects of ethanol extract of Zingiber officinale (Roscoe) rhizomes (Zingiberaceae) in mice and rats. Phytotherapy Research. 2006; 20 (9):764-772.

16. Ojewole JAO. Analgesic, anti-inflammatory and hypoglycemic effects of ethanol extract of Zingiber officinale (Roscoe) rhizomes (Zingiberaceae) in mice and rats. Phyto. Res. 2006; 20: 764-772

17. Priyanga Ranasinghe et al Cinnamomum zeylanicum (Ceylon cinnamon) as a potential pharmaceutical agent for type-2 diabetes mellitus: study protocol for a randomized controlled trial, September 2017, 18: 446

18. Jeyaraj S. Effect of jamun seed powder supplementation on the body mass index and fasting plasma glucose levels in woman with type 2 diabetes mellitus. Panacea J Health Sci 2012; 3:16-20.

19. Patel SS, Shah RS, Goyal R, Anti-hyperglycaemic, anti-hyperlipidemic and antioxidant effects of Dihar, a polyherbal ayurvedic formulation in streptozotocin induced diabetic rats.,Indian J Exp Biol. 2009 Jul; 47(7):564-70

20. Taher M, Fadzilah Adibah AM, Mohomad RS. A proanthocyanidin from Cinnamomum zeylanicum stimulates phosphorylation of insulin receptor in 3 T3-L1 adipocytes. J Teknologi. 2006; 44: 53-68.

21. Mandlik R.V., Desai S.K., Naik S.R., Sharma G., Kohli R.K. Antidiabetic activity of a polyherbal formulation (DRF/AY/5001) Indian J Exp Biol. 2008;46(8):599-606.

22. Khathi A, et al., Effects of Syzygium aromaticumderived triterpenes on postprandial blood glucose in streptozotocin-induced diabetic rats following carbohydrate challenge. PLoS One. 2013 Nov 22; 8(11): 81632

23. Agrawal P1, Rai V, Singh RB, Randomized placebo-controlled, single blind trial of holy basil leaves in patients with noninsulin-dependent diabetes mellitus., Int $\mathrm{J}$ Clin Pharmacol Ther. 1996Sep;34 (9):406-9

24. Subash Babu P, Prabuseenivasan S, Ignacimuthu S. Cinnamaldehyde--a potential antidiabetic agent. Phytomedicine. 2007; 14(1):15-22.

25. Ahmed AS, Ahmed Q, Saxena AK, and Jamal P. Evaluation of in vitro antidiabetic and antioxidant characterizations of Elettaria cardamomum (L.) Maton (Zingiberaceae), Piper cubeba L. f. (Piperaceae), and Plumeria rubra L. (Apocynaceae). Pak. J. Pharm. Sci. 2017; 30(1), $113-126$

26. Barros L, Heleno SA, Carvalho AM, Ferreira IC. System-atic evaluation of the antioxidant potential of different parts of Foeniculum vulgare Mill from Portugal. Food Chem Toxicol.2009; 47 (10):2458-64.

27. Krishnat S Yadav, Characterization of Anti-Diabetic Herbs \& Its Potential Agents in Indian Species: A Review, IOSR Journal of Dental and Medical Sciences 16(2, Version III) February 2017:70-78 •

28. Taibi DM, Landis CA, Petry $\mathrm{H}$ and Vitiello MV. (2007). A systematic review of valerian as a sleep aid: safe but not effective. Sleep Medicine Reviews, 11(3), 209 -30.

29. Usha Chakraborty and Hariswami Da, Antidiabetic and Antioxidant Activities of Cinnamomum tamala Leaf Extracts in Stz-Treated Diabetic Rats, Global Journal of Biotechnology \& Biochemistry 2010,5 (1): $12-18$

30. Doha Abdou Mohamed, Ibrahim Mohamed Hamed and Karem Aly Fouda ,Anti-oxidant and Antidiabetic Effects of Cumin Seeds Crude Ethanol Extract , Journal of Biological Sciences 2018 Volume 18(5): 251-259

31. D.K. Arulmozhi, R. Kurian, A. Veeranjaneyulu \& S.L. Bodhankar, Antidiabetic and Antihyperlipidemic Effects of Myristica fragrans. in Animal Models, journal of pharmaceutical biology, Oct 2008, Pages 64-68

32. Ahmed AS, Ahmed Q, Saxena AK, Jamal P.,Evaluation of in vitro antidiabetic and antioxidant characterizations of Elettaria cardamomum (L.) Maton (Zingiberaceae), Piper cubeba L. $f$. (Piperaceae), and Plumeria rubra L. (Apocynaceae), Pak J Pharm Sci. 2017 Jan;30(1):113-126

33. Purva MC, Meena MS. A review on role of Prakriti in aging. AYU 2011;32: 20-4

34. Govindarajan R, Pushpangadan P, Vijayakumar M. Anti-oxidant approach to disease management and role of rasayana herbs of Ayurveda. J Ethnopharmacol. 2005; 99: 165-78.

35. Rajasekharan S, Tuli SN. Vijayasara, Pterocarpus marsupium in the treatment of madhumeha(diabetes mellitus)-A Clinical trial. J Res Indian Med Yoga Homeo. 1976;11:9-14

36. Dhanabal SP, Suresh B, Kokate CK, Ramanathan M, Kumar EP. Hypoglycemic activity of Pterocarpus marsupium Roxb. Phytother Res. 2006; 20: 4-8.

37. Manickam M, Ramanathan M, Jahromi MA, Chansouria JP, Ray AB. Anti-hyperglycemic activity of phenolics from Pterocarpus marsupium. J Nat Prod. 1997; 60: 609-10.

38. Kidwai JR, Ahamad F, Khalid P, Khan MM, Chaubey M, Rastogi AK. Hypoglycemic activity of Pterocarpus marsupium wood. J Ethnopharmacol. 1991; 35:71

39. Arun N, Nalini N. Efficacy of turmeric on blood sugar and polyol pathway in diabetic albino rats.Plant Foods for Human Nutrition. 2002; 57 (1):41-52.

40. Murugan P, Pari L. Influence of tetrahydrocurcumin on hepatic and renal functional markers and protein levels in experimental type 2 diabetic rats. Basic 
and Clinical Pharmacology and Toxicology. 2007; 101 (4):241-245.

41. Wesom kooti et al., The role of medicinal plants in the treatment of diabetes: a systematic review, electron physician 2016;1832-1842

42. Sreelatha S, Inbavalli R (2012) Antioxidant, antihyperglycaemic, and antihyperlipidemic effects of Coriandrumsativum leaf and stem in alloxaninduced diabetic rats. J Food Sci 77:119-123.

43. Kar A, Choudhary BK, Bandyopadhyay NG. Comparative evaluation of hypoglycaemic activity of some Indian Medicinal Plants in alloxan diabetic rats. J Ethnopharmacol. 2003; 84: 105
44. Ahmed AS, Ahmed Q, Saxena AK, Jamal P.,Evaluation of in vitro antidiabetic and antioxidant characterizations of Elettaria cardamomum (L.) Maton (Zingiberaceae), Piper cubeba L. $f$. (Piperaceae), and Plumeria rubra $L$. (Apocynaceae), Pak J Pharm Sci. 2017 Jan; 30 (1):113-126

45. Doha Abdou Mohamed, Ibrahim Mohamed Hamed and Karem Aly Fouda, Anti-oxidant and Antidiabetic Effects of Cumin Seeds Crude Ethanol Extract, Journal of Biological Sciences 2018Volume 18 (5): 251-259. 